\title{
Somatostatin Treatment for Ectopic ACTH Syndrome due to Pancreatic Neuroendocrine Tumors: Review of the Literature
}

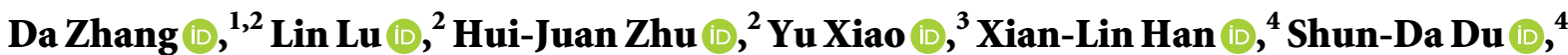 \\ Hua-Dan Xue $\mathbb{D},{ }^{5}$ Qing-Xing Liu $\mathbb{D},{ }^{6}$ Zhao-Hui Zhu $\mathbb{D},{ }^{6}$ Ming-Ming Hu $\mathbb{D},{ }^{2}$ Xiao Zhai $\mathbb{D},{ }^{2}$ \\ Xiao-Ping Xing $\mathbb{D},{ }^{2}$ and Zhao-Lin Lu $\mathbb{D}^{2}$ \\ ${ }^{1}$ Department of Endocrinology, Air Force Medical Center, People's Liberation Army, Beijing 100142, China \\ ${ }^{2}$ Department of Endocrinology, Key Laboratory of Endocrinology of National Health Commission, \\ Peking Union Medical College Hospital, Chinese Academy of Medical Science and Peking Union Medical College, \\ Beijing 100730, China \\ ${ }^{3}$ Department of Pathology, Peking Union Medical College Hospital, \\ Chinese Academy of Medical Science and Peking Union Medical College, Beijing 100730, China \\ ${ }^{4}$ Department of General Surgery, Peking Union Medical College Hospital, \\ Chinese Academy of Medical Science and Peking Union Medical College, Beijing 100730, China \\ ${ }^{5}$ Department of Radiology, Peking Union Medical College Hospital, \\ Chinese Academy of Medical Science and Peking Union Medical College, Beijing 100730, China \\ ${ }^{6}$ Department of Nuclear Medicine, Peking Union Medical College Hospital, \\ Chinese Academy of Medical Science and Peking Union Medical College, Beijing 100730, China \\ Correspondence should be addressed to Lin Lu; lulin88@sina.com
}

Received 2 July 2021; Revised 21 January 2022; Accepted 1 February 2022; Published 28 February 2022

Academic Editor: Giuseppe Reimondo

Copyright ( 2022 Da Zhang et al. This is an open access article distributed under the Creative Commons Attribution License, which permits unrestricted use, distribution, and reproduction in any medium, provided the original work is properly cited.

\begin{abstract}
Objectives. To analyze and summarize the effect of SSA treatment on EAS due to p-NETs (EAS-p-NETs). Methods. Thirteen patients with EAS-p-NETs treated with SSAs at our center or described in the literature were included in this study. Clinical characteristics, laboratory data, imaging studies, histopathologic results, the effect of SSA treatment, and the prognosis of these EAS-p-NET patients were evaluated. Results. Four males and 9 females with an average age of 42.9 years were included in the study. The mean duration of follow-up was $38.8 \pm 28.2$ months. As one of the combined treatment measures, SSAs controlled the levels of ACTH and cortisol in 9 of the 13 patients (69.2\%). Partial response was observed in 3 patients (23.1\%), stable disease in 2 patients $(15.4 \%)$, and progressive disease in 6 patients $(46.2 \%)$. The median time to tumor progression was 24 months, and the median overall survival was 61 months. The side effects of SSA treatment included temporary mild abdominal pain, diarrhea, gallstones, and cholecystitis. Conclusions. As a supplemental therapy, SSA treatment led to clinical and biochemical improvement with a good safety profile in patients exhibiting EAS-p-NET with metastasis. However, tumor progression was inhibited by SSA treatment in only a few patients. Combined with other treatments, SSAs may improve the prognosis of patients with EAS-p-NETs.
\end{abstract}

\section{Introduction}

Cushing's syndrome (CS) is a rare endocrine disease. The prevalence of CS ranges from 0.7 to 2.4 per million people per year [1]. CS is classified into adrenocorticotropic hormone (ACTH)-dependent and ACTH-independent causes.
Approximately $10-20 \%$ of CS cases are due to ectopic ACTH syndrome (EAS), which originates from an ACTH-producing or corticotropin-releasing hormone (CRH)-producing neuroendocrine tumor outside of the pituitary [2]. EAS has been reported to be due to small-cell carcinoma of the lung, bronchial carcinoid tumors, thymus carcinoid, 
pheochromocytoma, pancreatic neuroendocrine tumors ( $\mathrm{p}$ NETs), and gut carcinoids. p-NETs are present in up to $16 \%$ of EAS cases [3]. p-NETs are a group of heterogeneous diseases with varying tumor biology and clinical presentations. Unfortunately, p-NETs often metastasize widely by the time the clinical manifestations of CS appear. The overall 5year survival rate of patients with high-grade p-NETs does not exceed $40-60 \%[4,5]$.

The treatment of EAS from p-NETs with metastasis is quite challenging. The first goal is to control the excess production of ACTH or CRH and relevant clinical symptoms of hypercortisolism. Another goal is to control tumor progression. Somatostatin analogs (SSAs) are the first-line therapy for functional p-NETs, such as unresectable insulinoma, vasoactive intestinal polypeptide secreting tumors (VIPomas), and glucagonoma [6]. A meta-analysis systematically showed an antiproliferative effect of SSAs in advanced gastroenteropancreatic neuroendocrine tumors (GEP-NETs) [7]. In the management of GEP-NETs, stabilization of tumor growth, rather than tumor shrinkage, is the most frequent response to treatment. SSAs stabilize tumor growth in $36-70 \%$ of patients with metastatic GEP-NETs [8]. The rate of tumor progression in patients with pancreatic primary sites in the CLARINET study was $42.8 \%$ in the SSA group and $63.3 \%$ in the placebo group [9]. However, EAS is a rare manifestation in functional p-NETs. At present, there are few studies regarding the effect of SSAs in EAS due to p-NETs, and large-scale research is lacking. Thus, the effect of SSA treatment on EAS due to p-NETs should be evaluated.

In this article, we present a case of ectopic CRH syndrome stemming from a p-NET with liver metastasis successfully treated with octreotide long-acting release (LAR) and analyze the clinical characteristics and prognosis of 12 patients with EAS due to p-NETs treated with SSA in the literature to analyze and summarize the effect of SSA treatment on EAS due to p-NETs.

\section{Methods}

2.1. Medical Information of This Case. The medical records of the patient with an EAS-p-NET with multiple liver metastases at our hospital were reviewed. Information related to clinical characteristics, laboratory data, imaging studies, histopathologic results, treatment, and prognosis was collected. This study was approved by the Institutional Review Board of our hospital. Informed consent was obtained from the patient and her parents.

CS was diagnosed according to the 2008 clinical practice guidelines for the diagnosis of CS [10]. A level of morning ACTH persistently greater than $15 \mathrm{pg} / \mathrm{mL}$ almost always results from ACTH-dependent etiology [11]. Bilateral inferior petrosal sinus sampling (BIPSS) is recommended in cases with no detection of a visible pituitary adenoma on MRI, no suppressible high-dose dexamethasone suppression test (HDDST), and suspected ectopic lesions to differentiate the causes. A basal central : peripheral ratio of ACTH greater than $2: 1$ at baseline and/or greater than $3: 1$ after stimulation with CRH or desmopressin (DDAVP) is consistent with Cushing's disease, otherwise indicating a diagnosis of
EAS [12]. Subsequently, chest CT, abdominal CT, octreotide scanning, and PET/CT are carried out to detect the ectopic lesions individually.

The antitumor response to SSAs was assessed every 2-3 months using CT or MRI. The tumor response was graded according to the Response Evaluation Criteria in Solid Tumors (RECIST) version 1.1 [13]. Complete response (CR) refers to the disappearance of all target lesions. Partial response (PR) refers to at least a $30 \%$ decrease in the sum of the diameters of the target lesions. Progressive disease (PD) refers to at least a $20 \%$ increase in the sum of the diameters of the target lesions or the appearance of one or more new lesions. Stable disease (SD) refers to neither sufficient shrinkage to qualify for PR nor sufficient increase to qualify for PD. The median time to tumor progression (TTP) and median overall survival (OS) were calculated using the Kaplan-Meier method.

2.2. Literature Review. A systematic literature review was conducted by searching PubMed, Web of Science, and EMBASE for all relevant and available articles published in English. MeSH terms included "somatostatin/administration and dosage/analogs and derivatives/therapeutic use," "octreotide/administration and dosage/analogs and derivatives/therapeutic use," "ACTH syndrome, ectopic," "neuroendocrine tumors," and "pancreas" and "pancreatic." Original research, case reports, case series, or review articles published between 1950 and 2020 were included. Studies analyzing cases that were without somatostatin treatment or were not from p-NETs were all excluded.

\subsection{Whole-Exome Sequencing of the Blood and Tumor Tissue.} Whole-exome sequencing (WES) was performed using germline DNA samples and tumor DNA samples from our patient. DNA of peripheral blood leucocytes was captured using an Agilent SureSelect Human All Exon v6 (58 Mb) Kit (Agilent Technologies) and sequenced on an Illumina HiSeq 2000 (Illumina) with $150 \mathrm{bp}$ paired-end reads. Sequence reads were mapped to the hg19/GRCh37 human reference genome. Alignment and variant calling were performed using the Burrows-Wheeler Aligner (BWA)-MEM and the Genome Analysis Toolkit (GATK V.3.7.0), respectively, with default parameters. The number of total reads generated was 109 million, resulting in a median target coverage of $145 \times(97.64 \%$ of targets covered at $>20 x)$. For USP8, the median percent of bases covered at 20x was $100 \%$ for both coding exons. The output variant call format (VCF) files were annotated to identify and classify the disease-relevant variants using ANNOVAR software (https://www. openbioinformatics.org/annovar/) and ENSEMBL Variant Effect Predictor v91. All identified variants were annotated according to the guidelines published by the Human Genome Variation Society (HGVS). Bioinformatic analyses were used to rule out rare variants (present in $<0.5 \%$ of allele frequency) in known p-NET-associated genes (e.g., AIP, GNAS, PDE8B, MEN1, PRKAR1A, USP8, and SDHx). Targeted amplicon sequencing using DNA derived from peripheral blood leucocytes and Sanger sequencing using a 
3730XL autosequencer (Applied Biosystems) were used to confirm mutations identified by WES. Sequences were analyzed using SeqMan 7.0 (DNASTAR) software.

\section{Results}

3.1. Case Presentation. A 23-year-old female patient presented in April 2018 with a history of emotional changes, insomnia, increased appetite, weight gain, acne, fatigue (especially when climbing stairs), and irregular menstruation for 3 months, and she gradually developed Cushingoid manifestations with edema of the bilateral lower extremity and generalized fatigue. Initial laboratory evaluation revealed hypokalemia $(3.3 \mathrm{mmol} / \mathrm{L}$, normal range $3.5-5.5)$, hyperlipidemia, and impaired liver function. Her condition was also complicated with new onset diabetes and hypertension.

Endocrine workup showed that the morning serum cortisol level increased to $24.10 \mu \mathrm{g} / \mathrm{dL}$ (reference range: $4.0-22.3 \mu \mathrm{g} / \mathrm{dL})$, the midnight cortisol level increased to $35.23 \mu \mathrm{g} / \mathrm{dL}$, and the overnight low-dose (1 mg) dexamethasone suppression test was not suppressed. Elevated $24 \mathrm{~h}$ urinary free cortisol (UFC) excretion $(653.64 \mu \mathrm{g}$ per day; reference range: $12.3-103.5)$ was found. The morning plasma ACTH level was significantly increased to $139.0 \mathrm{pg} / \mathrm{mL}$ (reference range: $0-46 \mathrm{pg} / \mathrm{mL}$ ). No central-to-peripheral ACTH gradient was observed on BIPSS (the ratio of central/ peripheral ACTH was 1.12 at baseline and 1.20 after DDAVP stimulation). Abdomen high-resolution CT/MRI demonstrated multiple blood-rich nodules in the liver and a solid nodule $(1.1 \times 0.8 \mathrm{~cm})$ in the body-tail junction of the pancreas, with mild hyperplasia of adrenals (Figure 1). Somatostatin receptor scintigraphy (SRS) and $18 \mathrm{~F}-\mathrm{FDG}-\mathrm{PET} / \mathrm{CT}$ revealed multiple metastatic foci of high uptake in the liver with a standardized uptake value (SUV) of 4.2-6.6 but no uptake of pancreatic lesions. ${ }^{68} \mathrm{Ga}-\mathrm{DOTATOC}$ PET/CT was performed further, showing increased uptake in both metastatic nodules in the liver and primary pancreas. The maximum SUV of the pancreatic tumor was 20.7 and that of the liver metastases was 21.4-26.6. Histological examination after biopsy demonstrated the presence of a p-NET and liver metastases with positive immunostaining for chromogranin A and synaptophysin. The pancreatic tumor and liver metastases had a Ki-67 index of $15 \%$ and $10 \%$, respectively. Interestingly, the tumors showed positive immunohistochemical staining for $\mathrm{CRH}$ but negative staining for ACTH (Figure 2).

Short-term (13 days) octreotide therapy $(100 \mu \mathrm{g}$ subcutaneously, q8h) prompted marked reductions in the ACTH level from 131 to $63 \mathrm{pg} / \mathrm{mL}$ and in the cortisol level from 57.47 to $7.16 \mu \mathrm{g} / \mathrm{dL}$, and the UFC level decreased to the normal range ( $55 \mu \mathrm{g}$ per day). The recommended treatment for this patient was octreotide LAR at a dose of $20 \mathrm{mg}$ every 28 days for 7 cycles, leading to a favorable clinical and biochemical response. The hormonal tests were maintained in the normal range with the ACTH level ranging between 23.0 and $53.4 \mathrm{ng} / \mathrm{L}$ and the UFC level ranging between 6.96 and $48.84 \mu \mathrm{g} / 24 \mathrm{~h}$. During the first two weeks, the patient experienced transient mild abdominal pain and diarrhea. After 7 cycles of somatostatin LAR treatment, abdominal
MRI showed that the primary and metastatic tumors remained stable. However, the enhanced intensity of the lesions was reduced on contrast MRI, indicating that the blood supply of the lesions had decreased (Figure 1). ${ }^{18} \mathrm{~F}$ FDG PET-CT also demonstrated slightly decreased radioactivity uptake in the liver metastases, with SUVmax values of 3.5-6.0, and ${ }^{68} \mathrm{Ga}$-DOTATOC PET-CT revealed that the SUVmax in the liver foci decreased to 17.1-20.2, while the SUVmax in the pancreatic foci decreased to 11.6.

The patient underwent laparoscopic-guided body-caudal pancreatectomy plus splenectomy after the 8th cycle injection of octreotide LAR. Radiofrequency ablation combined with resection of multiple hepatic metastases was conducted 3 weeks later. The Ki-67\% indices of the pancreatic tumor and liver metastases were $10 \%$ and $4 \%$, respectively. Immunostaining of somatostatin receptor 2 (SSTR2) was carried out to show positive results. Octreotide LAR treatment was continued for 24 cycles, and the patient received regular followup, showing long-term normalization of the levels of ACTH, cortisol, and $24 \mathrm{~h}$ UFC (Figure 3 ). The only side effect was mud-like gallstones suggested by ultrasound, without any symptoms. In October 2020, abdominal MRI and ${ }^{68} \mathrm{Ga}-$ DOTATOC PET/CT revealed some new liver metastatic lesions, with an SUVmax of 11.5. The patient underwent peptide receptor radionuclide therapy (PRRT) 6 times, once every 3 months, and received chemotherapy with capecitabine and temozolomide starting in October 2020. The liver metastatic foci diminished after PPRT and chemotherapy.

3.2. Literature Review. Twelve references were included that involved patients with EAS due to p-NETs, and all the references were published as case reports. There were 12 patients ( 4 male and 8 female) with EAS originating from p-NETs who were treated with SSA as one of the therapeutic strategies described in these reports (Table 1). The mean age at diagnosis was $42.9 \pm 13.2$ years. All the patients had typical Cushingoid manifestations. Ten of the 12 patients had hypokalemia with a mean value of $2.56 \pm 0.57 \mathrm{mmol} / \mathrm{L}$ (range from 1.7 to $3.8 \mathrm{mmol} / \mathrm{L}$ ). Ten patients were complicated with diabetes, and 8 patients had hypertension. The mean morning ACTH level was $269.6 \pm 183.8 \mathrm{pg} / \mathrm{mL}$ (range from 68.1 to $735 \mathrm{ng} / \mathrm{L}$ ). The median morning cortisol and UFC levels were $40 \mu \mathrm{g} / \mathrm{dL}$ (range from 15.2 to $1198 \mu \mathrm{g} / \mathrm{dL}$ ) and $986.5 \mu \mathrm{g} /$ day (range from 86.1 to $38,200 \mu \mathrm{g} / \mathrm{day}$ ), respectively. The size of the primary pancreatic tumor ranged from 1.2 to $6.0 \mathrm{~cm}(3.7 \pm 2.0 \mathrm{~cm})$. All the patients had metastasis at diagnosis. Five of the 12 patients had lymph node metastases, and 10 of the 12 patients had liver metastases. The median Ki-67 index of the pancreatic or metastatic tumors was $10.7 \%(<1 \%$ to $20 \%)$. Immunostaining for SSTR2 was performed in 2 patients, both of which were positive. Immunostaining for SSTR5 in liver metastatic lesions was performed in 1 patient, and the tumor was positively stained.

3.3. SSA Effects in Patients with EAS due to p-NETs. We evaluated our reported case together with the 12 cases from the literature review to analyze the effect of SSA in controlling hormone production and tumor growth. 


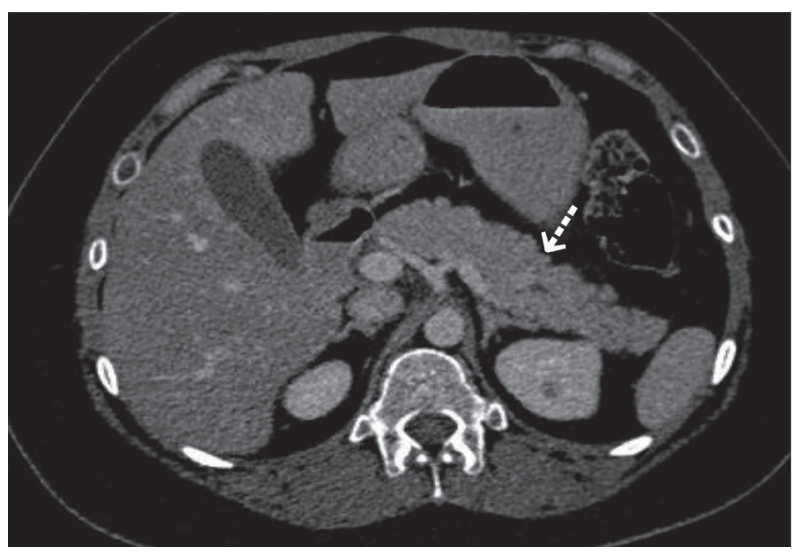

(a)

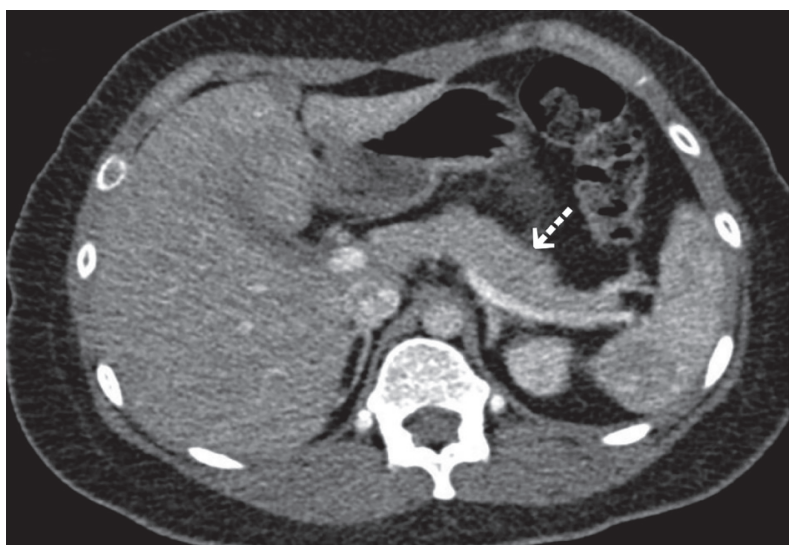

(c)

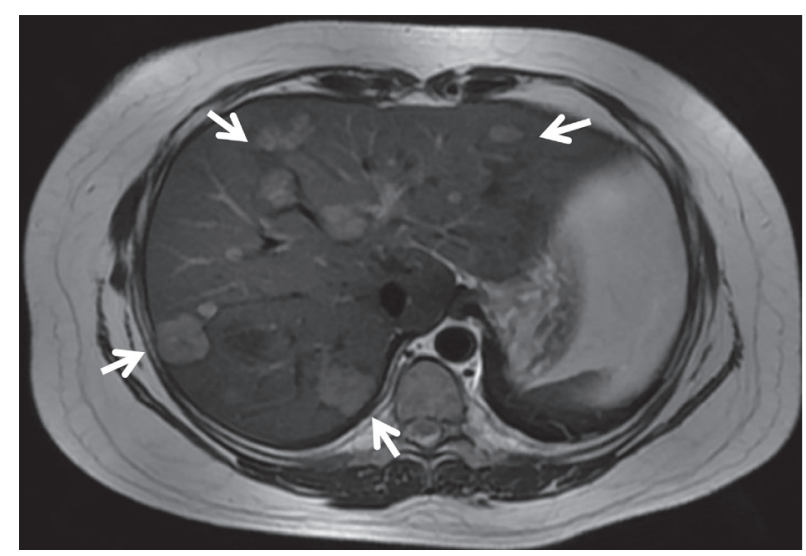

(b)

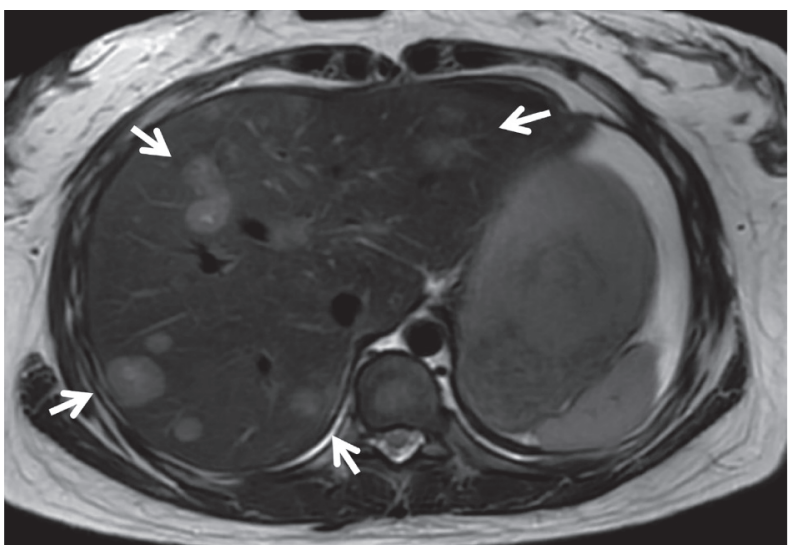

(d)

FIgURE 1: Abdominal CT and MRI images before (a and c) and after 7 cycles of octreotide LAR therapy (b and d) (case 1). (a) The abdominal CT image demonstrated a solid nodule in the body-tail junction of the pancreas (dotted arrow). (b) The abdominal MRI demonstrated multiple blood-rich nodules in the liver (arrow). (c) The abdominal CT image demonstrated that the pancreatic solid nodule was unclear after 7 cycles of octreotide LAR therapy (dotted arrow). (d) The abdominal MRI demonstrated that the sizes and numbers of hepatic metastatic nodules remained stable (arrow). CT: computed tomography. MRI: magnetic resonance imaging.

3.3.1. The Effect of SSA on the Excess Production of Hormones. The effect of controlling hypercortisolism after SSA treatment was recorded in 12 patients except patient 4 . ACTH and cortisol levels decreased to normal ranges after SSA therapy alone in 8 of those 12 patients (66.7\%). Combined with metyrapone, SSA effectively decreased the ACTH and cortisol levels in 1 patient. The types of SSA included octreotide LAR (20 mg per month), lanreotide, and octreotide $(50 \mu \mathrm{g} / \mathrm{d}$ to $1500 \mu \mathrm{g} / \mathrm{d})$. ACTH and cortisol were normalized in 8 patients who exhibited a good response in the octreotide loading test. The effect of decreasing the ACTH and cortisol levels appeared a few days after the onset of SSA application and was sustained for an average of 11.2 months (range: 2-24 months).

3.3.2. The Effect of SSA on Tumor Growth and Prognosis. The effect on tumor growth after SSA treatment was available in 11 patients with a mean follow-up time of $38.8 \pm 28.2$ months. Primary or metastatic tumor sizes decreased in 3 patients $(27.3 \%)$, remained stable in 2 patients $(18.2 \%)$, and progressed in size or number in 6 patients $(54.5 \%)$. The prognosis was mentioned for 7 patients. The median TTP was 24 months, and the median OS was 61 months. Four patients died of tumor progression or complications, including intestinal perforation, ARDS, and severe hepatic insufficiency. One patient remained clinically stable after chemotherapy, another patient planned to undergo radical surgical treatment, and our patient underwent PRRT.

3.3.3. Side Effect of SSA Treatment. The side effects of SSA were available for 7 patients. No side effects were recorded for 3 patients. Temporary mild abdominal pain, diarrhea, gallstones, and cholecystitis occurred in 4 patients. Additional glucocorticoid supplementation was not reported for any of the patients, even those in whom the ACTH and cortisol levels decreased rapidly. There were no treatment delays or interruptions due to adverse events.

\section{Results of WES}

In the present case, WES of the patient's peripheral blood and tumor tissue was carried out to investigate any genetic changes. A heterozygous variant of the USP8 gene (p.P750A) 


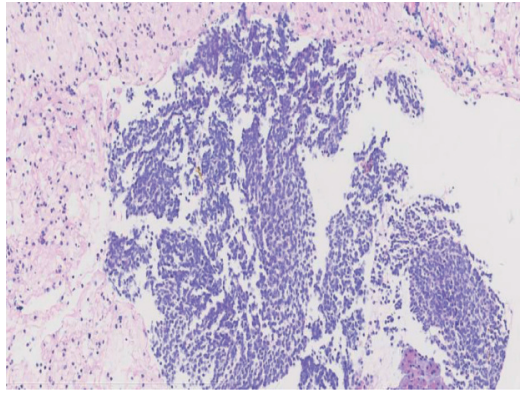

(a)

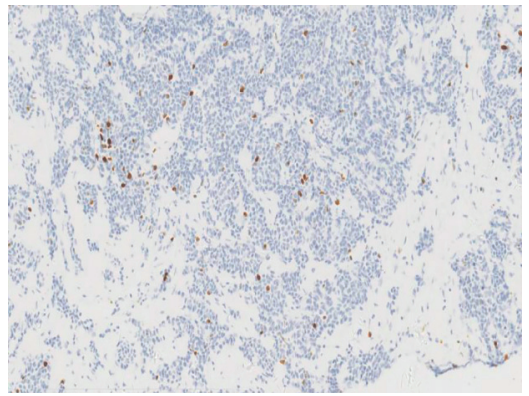

(d)

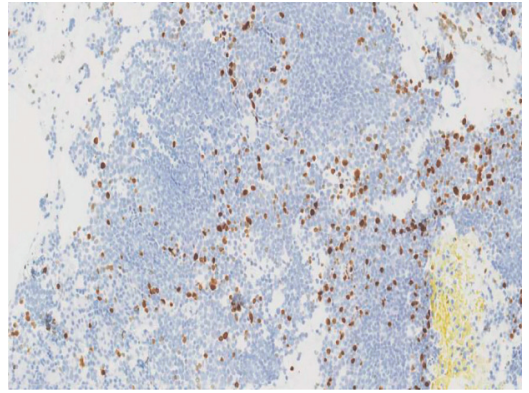

(b)

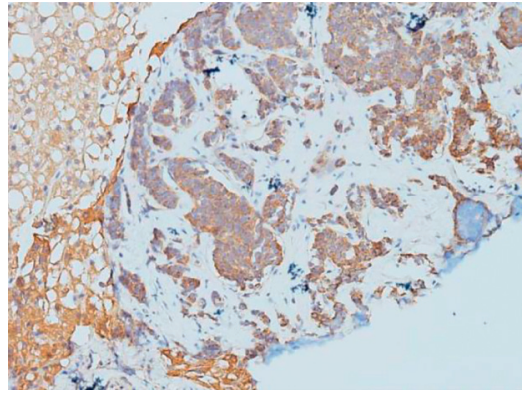

(e)

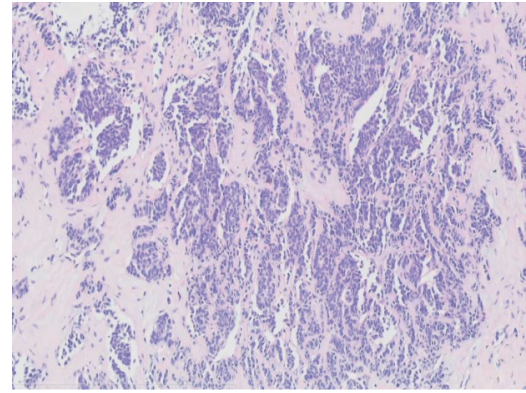

(c)

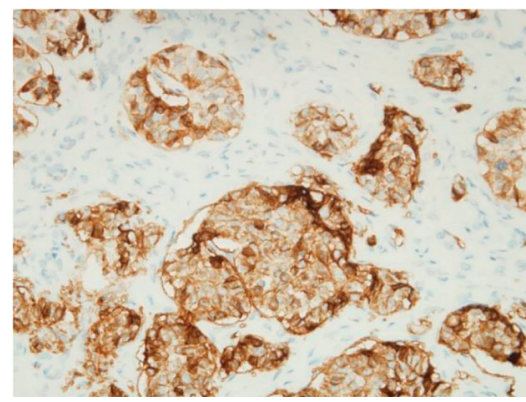

(f)

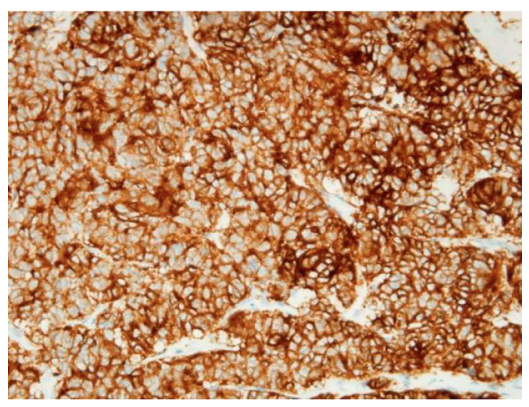

(g)

Figure 2: Pathology and immunochemistry staining of tumors of the pancreas (a, b, e, and f) and liver metastasis (c, d, and g). (a) Pancreatic neuroendocrine tumor (hematoxylin and eosin staining, magnification $\times 200$ ); (b) the percentage of Ki-67-positive cells (brown color) was $15 \%$ in the pancreatic tumor (magnification $\times 200$ ); (c) liver metastases of the neuroendocrine tumor (hematoxylin and eosin staining, magnification $\times 200$ ); (d) the percentage of Ki-67-positive cells (brown color) was $10 \%$ in the hepatic metastases (magnification $\times 200)$; $(\mathrm{e})$ positive immunostaining for CRH (brown color) in the liver metastasis (magnification $\times 200$ ); (f) \& (g) positive immunostaining for SSTR2 (brown color) in the pancreatic tumor (magnification $\times 400$ ) (f) and in the hepatic metastases (magnification $\times 400)(\mathrm{g})$.

inherited from her mother with unclear clinical significance was detected. Other related abnormalities were absent.

\section{Discussion}

Functional p-NETs are rare, representing approximately $1 \%$ of pancreatic neoplasia [26]. Ectopic ACTH-producing tumors are very rare types of functional p-NETs, with few reports in the literature and no consensus guidelines for management. Retrospective studies support the use of octreotide LAR in functioning and nonfunctioning lowgrade $\mathrm{p}$-NETs but not in p-NETs secreting ACTH or CRH. Hence, this study analyzed and summarized the effect of SSA treatment on EAS due to $\mathrm{p}$-NETs to provide evidence for this rare situation.

The inhibited secretion of peptides from the NET by SSA is mediated mainly through SSTR2 and SSTR5. SSTR2 is expressed in almost $80 \%$ of p-NETs [27]. SRS and ${ }^{68} \mathrm{Ga}-$ DOTATOC PET-CT can provide not only a better overall detection rate for diagnosis but also indications regarding SSTR expression on target lesions, allowing a decision regarding SSA treatment [28]. In addition, an in vivo octreotide loading test or immunostaining with SSTR2 and SSTR5 in tumor tissues may help to further predict the therapeutic response of SSA [29]. The positive octreotide loading test predicted the good effect of SSA in 7 patients in this case series. However, negative SRS or ${ }^{68} \mathrm{Ga}$-DOTATOC PET-CT could not determine whether there was no effect of octreotide treatment. Initiating therapy with octreotide $50-100 \mu \mathrm{g}$ s.c. for 7-10 days twice to thrice per day is recommended for gastroenteric NETs [30].

Although the expression of SSTR2 and SSTR5 has predictive value for SSA effects, notably, not all p-NET tissues from a different focus in a patient express similar 


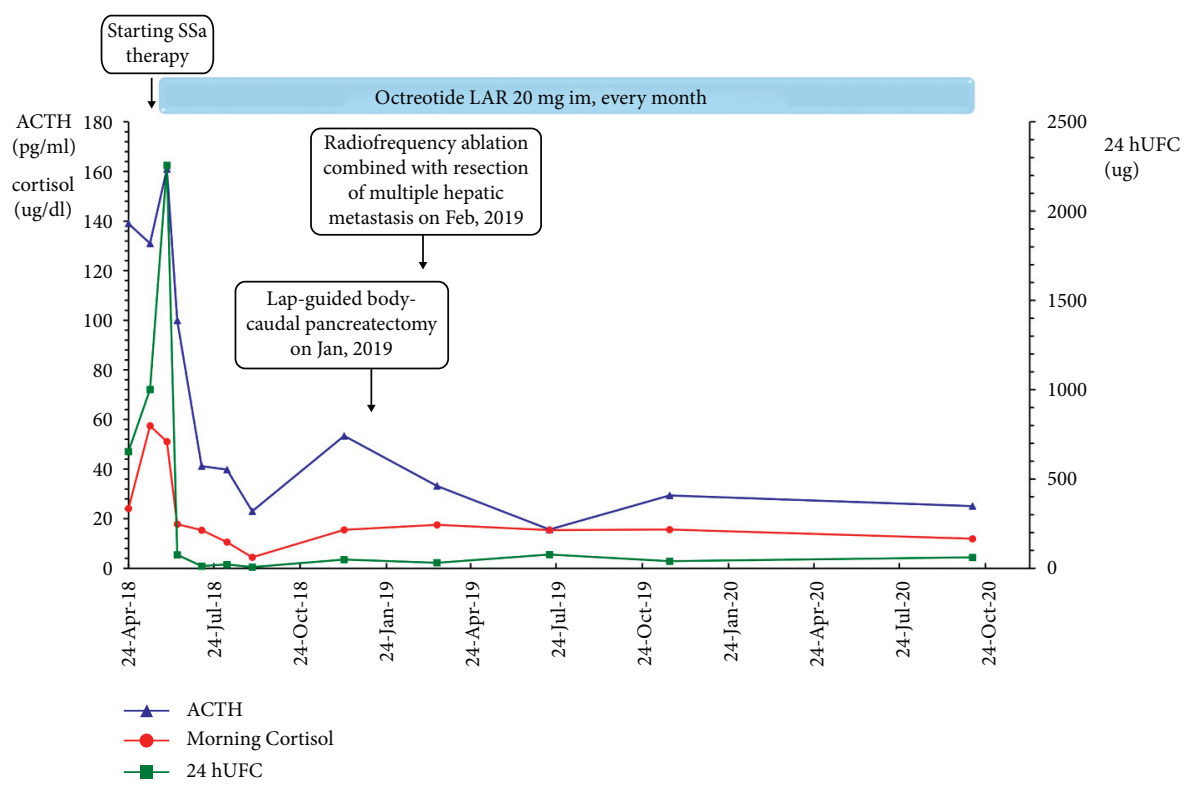

FIGURE 3: ACTH, morning cortisol, and UFC trends during the follow-up after SSA treatment and surgery. Short-term (13 days) octreotide therapy prompted marked reductions in ACTH levels (from 131 to $63 \mathrm{pg} / \mathrm{mL}$ ) and cortisol levels (from 57.47 to $7.16 \mu \mathrm{g} / \mathrm{dL}$ ), and UFC levels decreased to the normal range ( $55 \mu \mathrm{g}$ per day). Surgery and long-term octreotide LAR therapy maintained the hormones normally, with ACTH fluctuating from 23.0 to $53.4 \mathrm{ng} / \mathrm{L}$ and UFC fluctuating from 6.96 to $48.84 \mu \mathrm{g} / 24 \mathrm{~h}$.

subtypes of SSTRs. Therefore, each of the metastatic tumors might have a differential expression of SSTR subtypes [19]. The heterogeneity and pluripotency of p-NETs explain why some tumors respond well to SSAs while others either show no response or become resistant to treatment within months. In our study, the expression of SSTR2 or SSTR 5 was detected in cases 1,7 , and 8 , in which SSA had good effects on the control of hypercortisolism. The longest period of symptom control was 28 months. The potential mechanisms of nonresponsiveness may include downregulation of SSTRs or the outgrowth of clones lacking specific SSTR expression [31].

SSAs are generally recommended as a first-line treatment to slow disease progression in patients with advanced p-NETs by the North American Neuroendocrine Tumor Society because of the lack of toxicity of these drugs and the fact that they are well tolerated [32]. In a review of 15 studies including 481 patients with functional GEP-NETs, octreotide LAR achieved symptomatic relief in $74.2 \%$ of the patients and a biochemical response in 51.4\% [33]. EAS-pNETs had a total control rate of $75 \%$ in terms of symptomatic and biochemical control under first-line therapy with an SSA. The side effects of the SSA were mild [34]. However, some patients may develop gallstones after long-term treatment. According to our data, SSAs may be chosen as the first-line preoperative therapy in patients with EAS-p-NETs.

Apart from symptom control, SSAs also have the potential to control tumor growth. Among all 13 patients with EAS-p-NETs, tumor progression was controlled in 5 (38.5\%). Three patients (23.1\%) exhibited tumor progression during SSA therapy, although ACTH production was well controlled by SSA treatment. The median TTP for Ki$67>10 \%$ was 4 months, that for Ki- $67<5 \%$ was 15 months, and that for Ki-67 5-10\% was 12 months [35]. Octreotide treatment has been reported to stabilize tumor growth in $36.5 \%$ of metastatic endocrine GEP-NETs for at least 12 months without causing tumor regression [36]. PR and SD of tumor growth have been demonstrated in up to $10 \%$ and approximately $50 \%$ of functional GEP-NET patients [37]. In one Korean study, PR and SD were achieved in $2.2 \%$ and $88.9 \%$ of p-NET patients, respectively. The median TTP was 16.4 months (95\% confidence interval, 9.5-23.3 months) [34]. Jann et al [35] retrospectively analyzed the records of 43 patients with p-NETs (19 functional tumors without EAS-pNETs, 24 nonfunctional tumors) treated with octreotide LAR. After 12 months of SSA therapy, there was a total disease control rate of $42 \%$, including PR in $4.7 \%$ and SD in $37.2 \%$ of patients when evaluating tumor growth. The median OS for SSA responders (SD + PR) was 137 months versus 68 months for nonresponders (PD). The median TTP was 22 months for SSA responders and 3 months for nonresponders. Our data indicated that PR was achieved in $23.1 \%$ of the patients $(3 / 13)$ and that SD was achieved in $15.4 \%$ of the patients (2/13), with a median TTP of 24 months and a median OS of 61 months in patients with EASp-NETs. The antiproliferative effect of SSAs exhibited a disparity in different studies of GEP-NETs. However, previous studies have not evaluated the efficacy of SSAs in controlling tumor growth in EAS-p-NETs. In this study, the relatively low rate of tumor proliferation control by SSA may have been found because $66.7 \%$ of the patients had pathological features with $\mathrm{Ki}-67 \% \geq 10 \%$ at baseline, which might have a poor response to SSA, as in previous reports.

Considering the prognosis, grading of NETs, distant metastases, and severity of hypercortisolism may affect the prognosis of EAS patients [38]. The five-year survival rate was $78 \%$ without and $27 \%$ with distant metastases [39]. In our series, all the EAS-p-NET patients had distant metastases 


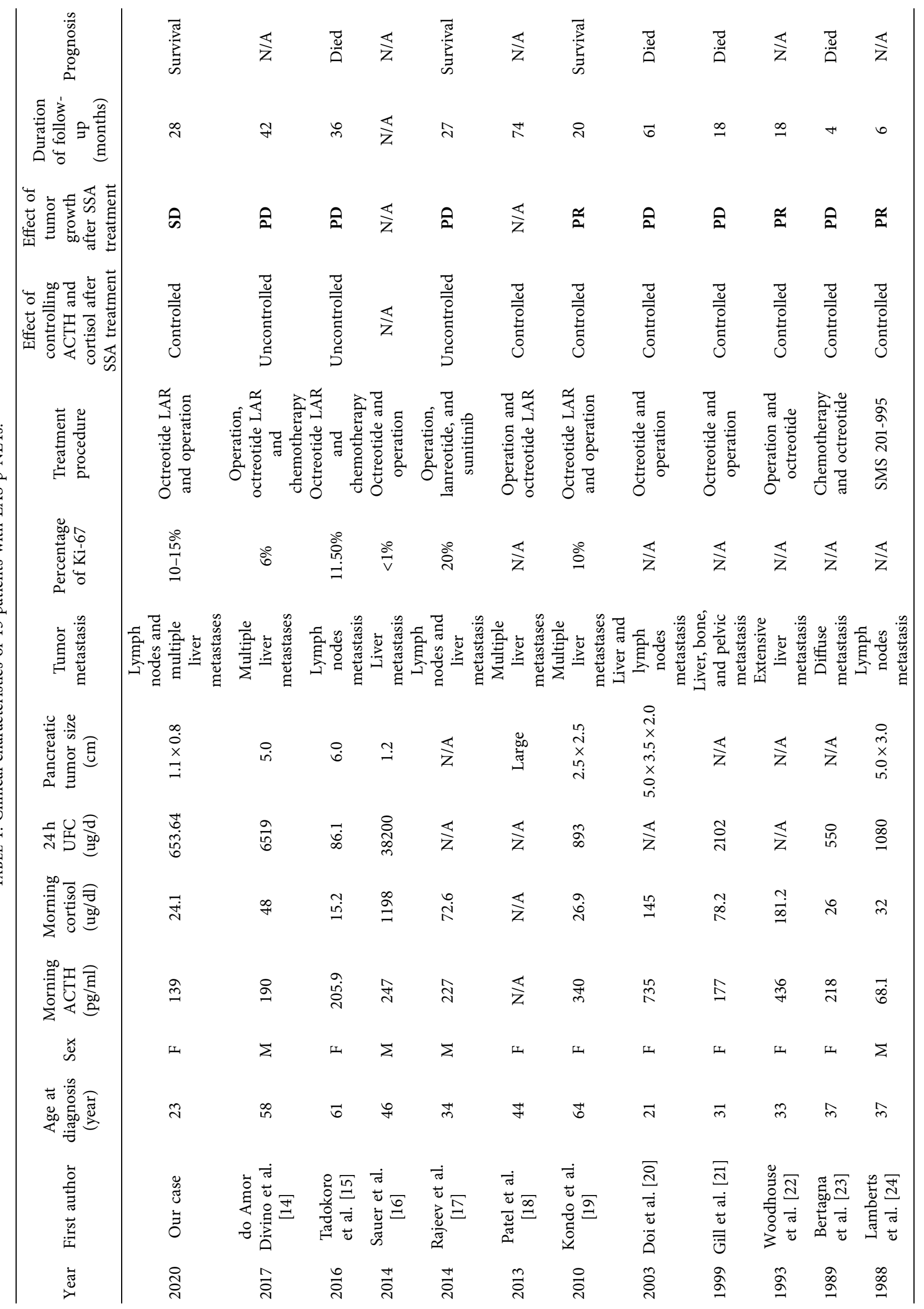




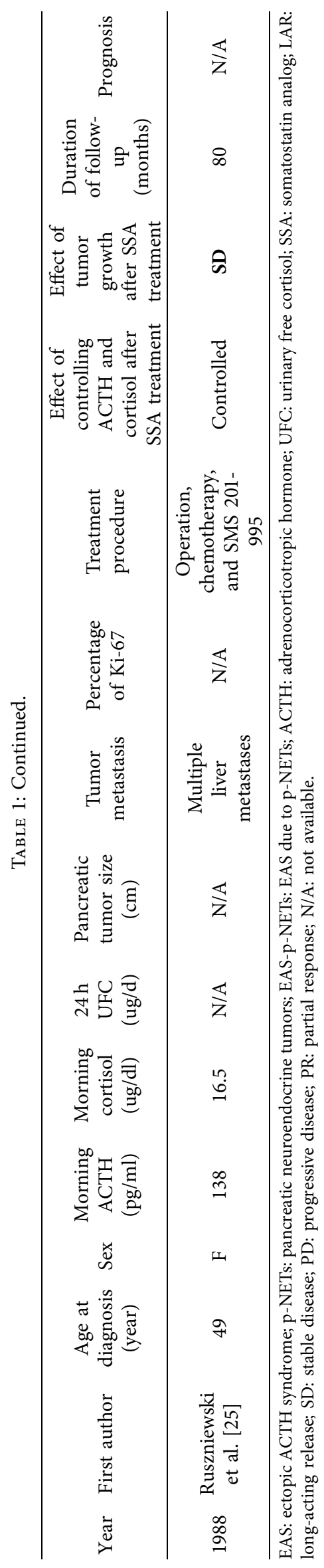


at the time of diagnosis. A multidisciplinary approach should be adopted to improve the prognosis of patients. Surgery with curative intent always has to be considered in grade 1 and 2 NETs, even if liver metastases are present [40]. P-NET patients with distant metastatic disease who underwent surgical resection of the primary tumor showed significantly better survival [39]. Patients with EAS who underwent surgery had a better survival rate than those who did not (5-year OS $85 \%$ vs. $51 \%, P<0.001$ ) [38]. Successful control of hypercortisolemia with SSAs provides an opportunity for further surgery and markedly reduces the operative and perioperative risk since EAS patients often have a high risk of fatal opportunistic infection, severe hypopotassemia, hyperglycemia, and hypertension. Thus, resection of the primary tumor and debulking surgery for metastases should be considered after controlling hypercortisolemia by SSA to improve the prognosis of EAS from p-NETs.

There are some limitations in this study. Initially, this was a retrospective study with a small sample size, as EAS-pNETs are quite rare, and the effect of tentative treatment with an SSA may be variable. As follow-up in most cases was rather short, the duration of the possible antiproliferative action of SSAs is unknown. In addition, not all patients underwent immunostaining of SSTRs, octreotide loading tests, or radiolabeled molecular imaging tests. Future research is required to assess the long-term efficacy of SSAs and improve the prognosis of EAS-p-NETs.

In summary, EAS-p-NETs are rare endocrinological entities associated with severe hypercortisolism and are difficult to manage since metastasis is common at diagnosis. Diagnostic imaging with SRS and 68 Ga-DOTATOC PETCT and immunostaining with SSTR2 provide information on SSTR expression status in the tumor, which combined with an octreotide loading test helps to predict the response to SSA treatment. SSAs may be used as first-line therapy before surgery and can lead to both instant clinical improvement with a good safety profile and control of tumor growth in some patients with EAS-p-NETs. SSAs could ameliorate severe conditions in patients and give them the chance to undergo subsequent surgery to improve their prognosis.

\section{Data Availability}

The data used in the study are available upon request to the corresponding author.

\section{Conflicts of Interest}

The authors declare that they have no conflicts of interest.

\section{Acknowledgments}

The authors are grateful to Jun Jiang of Beijing Macro \& Microtest Bio-Tech Co., Ltd., for providing the WES assay, CAMS Innovation Fund for Medical Science (CAMS-2017I2M-1-011), and the National Key Research and Development Program of China (no. 2016YFC0901500)

\section{Supplementary Materials}

Supplement Table 1: symptoms and hormone levels of 13 patients with EAS-p-NETs. Supplement Table 2: tumor characteristics of 13 patients with EAS-p-NETs. Supplement Table 3: treatment and prognosis of 13 patients with EAS-pNETs. (Supplementary Materials)

\section{References}

[1] J. Lindholm, S. Juul, J. O. L. Jørgensen et al., "Incidence and late prognosis of cushing's syndrome: a population-based study," Journal of Clinical Endocrinology \& Metabolism, vol. 86, no. 1, pp. 117-123, 2001.

[2] A. R. Hayes and A. B. Grossman, "The ectopic adrenocorticotropic hormone syndrome: rarely easy, always challenging," Endocrinology and Metabolism Clinics of North America, vol. 47, no. 2, pp. 409-425, 2018.

[3] K. Öberg, "Management of functional neuroendocrine tumors of the pancreas," Gland Surgery, vol. 7, no. 1, pp. 20-27, 2018.

[4] J. C. Yao, M. Hassan, A. Phan et al., "One hundred years after "carcinoid": epidemiology of and prognostic factors for neuroendocrine tumors in 35,825 cases in the United States," Journal of Clinical Oncology, vol. 26, no. 18, pp. 3063-3072, 2008.

[5] G. Rindi, M. Falconi, C. Klersy et al., "TNM staging of neoplasms of the endocrine pancreas: results from a large international cohort study," Journal of the National Cancer Institute, vol. 104, no. 10, pp. 764-777, 2012.

[6] M. Pavel, D. O'Toole, F. Costa et al., "ENETS consensus guidelines update for the management of distant metastatic disease of intestinal, pancreatic, bronchial neuroendocrine neoplasms (NEN) and NEN of unknown primary site," Neuroendocrinology, vol. 103, no. 2, pp. 172-185, 2016.

[7] E. Merola, F. Panzuto, and G. D. Fave, "Antiproliferative effect of somatostatin analogs in advanced gastro-entero-pancreatic neuroendocrine tumors: a systematic review and metaanalysis," Oncotarget, vol. 8, no. 28, pp. 46624-46634, 2017.

[8] R. Arnold, B. Simon, and M. Wied, "Treatment of neuroendocrine GEP tumours with somatostatin analogues: a review," Digestion, vol. 62, no. 1, pp. 84-91, 2000.

[9] M. E. Caplin, M. Pavel, J. B. Cwikła et al., "Lanreotide in metastatic enteropancreatic neuroendocrine tumors," New England Journal of Medicine, vol. 371, no. 3, pp. 224-233, 2014.

[10] L. K. Nieman, B. M. K. Biller, J. W. Findling et al., "The diagnosis of cushing's syndrome: an endocrine society clinical practice guideline," Journal of Clinical Endocrinology \& Metabolism, vol. 93, no. 5, pp. 1526-1540, 2008.

[11] C. Invitti, F. P. Giraldi, M. de Martin, and F. Cavagnini, "Diagnosis and management of cushing's syndrome: results of an Italian multicentre study," Journal of Clinical Endocrinology \& Metabolism, vol. 84, no. 2, pp. 440-448, 1999.

[12] E. H. Oldfield, J. L. Doppman, L. K. Nieman et al., "Petrosal sinus sampling with and without corticotropin-releasing hormone for the differential diagnosis of cushing's syndrome," New England Journal of Medicine, vol. 325, no. 13, pp. 897-905, 1991.

[13] E. A. Eisenhauer, P. Therasse, J. Bogaerts et al., "New response evaluation criteria in solid tumours: revised RECIST guideline (version 1.1)," European Journal of Cancer, vol. 45, no. 2, pp. 228-247, 2009. 
[14] P. H. do Amor Divino, K. R. Marchetti, M. Q. Almeida, and R. P. Riechelmann, "Functional pancreatic neuroendocrine tumour causing cushing's syndrome: the effect of chemotherapy on clinical symptoms," Ecancermedicalscience, vol. 11, p. 773, 2017.

[15] R. Tadokoro, S. Sato, F. Otsuka et al., "Metastatic pancreatic neuroendocrine tumor that progressed to ectopic adrenocorticotropic hormone (ACTH) syndrome with growth hormone-releasing hormone (GHRH) production," Internal Medicine, vol. 55, no. 20, pp. 2979-2983, 2016.

[16] N. Sauer, C. S. z. Wiesch, J. Flitsch et al., "Cushing's syndrome due to a corticotropin-releasing hormone- and adrenocorticotrophic hormone-producing neuroendocrine pancreatic tumor," Endocrine Practice, vol. 20, no. 4, pp. e53-e57, 2014.

[17] S. P. Rajeev, S. McDougall, M. Terlizzo, D. Palmer, C. Daousi, and D. J. Cuthbertson, "Evolution in functionality of a metastatic pancreatic neuroendocrine tumour (pNET) causing cushing's syndrome: treatment response with chemotherapy," BMC Endocrine Disorders, vol. 14, no. 1, p. 70, 2014.

[18] F. B. Patel, S. Khagi, K. P. Daly, R. M. Lechan, V. Ummaritchot, and M. W. Saif, "Pancreatic neuroendocrine tumor with ectopic adrenocorticotropin production: a case report and review of literature," Anticancer Research, vol. 33, no. 9, pp. 4001-4005, 2013.

[19] T. Kondo, R. Matsuyama, H. Ashihara et al., "A case of ectopic adrenocorticotropic hormone-producing pancreatic neuroendocrine tumor with multiple liver metastases," Endocrine Journal, vol. 57, no. 3, pp. 229-236, 2010.

[20] M. Doi, T. Imai, M. Shichiri et al., "Octreotide-sensitive ectopic ACTH production by islet cell carcinoma with multiple liver metastases," Endocrine Journal, vol. 50, no. 2, pp. 135-143, 2003.

[21] G. V. Gill, A. Yong, E. Power, and J. Ramage, "Carcinoidassociated ectopic ACTH syndrome with variable response to octreotide," Postgraduate Medical Journal, vol. 75, no. 880, pp. 98-101, 1999.

[22] N. J. Y. Woodhouse, S. Dagogo-Jack, M. Ahmed, and R. Judzewitsch, "Acute and long-term effects of octreotide in patients with ACTH-dependent cushing's syndrome," The American Journal of Medicine, vol. 95, no. 3, pp. 305-308, 1993.

[23] X. Bertagna, C. Favrod-Coune, H. Escourolle et al., "Suppression of ectopic adrenocorticotropin secretion by the longacting somatostatin analog octreotide," Journal of Clinical Endocrinology \& Metabolism, vol. 68, no. 5, pp. 988-991, 1989.

[24] S. W. J. Lamberts, H. W. Tilanus, A. I. J. Klooswijk, H. A. Bruining, A. J. V. D. Lely, and F. H. D. Jong, "Successful treatment with SMS 201-995 of cushing's syndrome caused by ectopic adrenocorticotropin secretion from a metastatic gastrin-secreting pancreatic islet cell carcinoma," Journal of Clinical Endocrinology \& Metabolism, vol. 67, no. 5, pp. 1080-1083, 1988.

[25] P. Ruszniewski, F. Girard, R. Benamouzig, M. Mignon, and S. Bonfils, "Long acting somatostatin treatment of paraneoplastic cushing's syndrome in a case of Zollinger-Ellison syndrome," Gut, vol. 29, no. 6, pp. 838-842, 1988.

[26] J. Hallet, C. H. L. Law, M. Cukier, R. Saskin, N. Liu, and S. Singh, "Exploring the rising incidence of neuroendocrine tumors: a population-based analysis of epidemiology, metastatic presentation, and outcomes," Cancer, vol. 121, no. 4, pp. 589-597, 2015.

[27] M. Papotti, M. Bongiovanni, M. Volante et al., "Expression of somatostatin receptor types 1-5 in 81 cases of gastrointestinal and pancreatic endocrine tumors. A correlative immunohistochemical and reverse-transcriptase polymerase chain reaction analysis," Virchows Archiv, vol. 440, no. 5, pp. 461-475, 2002.

[28] F. Sanguin, N. Albiger, C. Betterle et al., "Diagnostic and therapeutic challenge in the management of a patient with ectopic adrenocorticotropin secretion," Journal of Endocrinological Investigation, vol. 33, no. 7, pp. 507-508, 2010.

[29] D. Kaemmerer, L. Peter, A. Lupp et al., "Molecular imaging with $68 \mathrm{Ga}$-SSTR PET/CT and correlation to immunohistochemistry of somatostatin receptors in neuroendocrine tumours," European Journal of Nuclear Medicine and Molecular Imaging, vol. 38, no. 9, pp. 1659-1668, 2011.

[30] B. Eriksson, G. Klöppel, E. Krenning et al., "Consensus guidelines for the management of patients with digestive neuroendocrine tumors - well-differentiated jejunal-ileal tumor/carcinoma," Neuroendocrinology, vol. 87, no. 1, pp. 8-19, 2008.

[31] W. W. de Herder, "When and how to use somatostatin analogues," Endocrinology and Metabolism Clinics of North America, vol. 47, no. 3, pp. 549-555, 2018.

[32] T. R. Halfdanarson, J. R. Strosberg, L. Tang et al., "The North American neuroendocrine tumor society consensus guidelines for surveillance and medical management of pancreatic neuroendocrine tumors," Pancreas, vol. 49, no. 7, pp. 863-881, 2020.

[33] I. M. Modlin, M. Pavel, M. Kidd, and B. I. Gustafsson, "Review article: somatostatin analogs in the treatment of gastroentero-pancreatic neuroendocrine (carcinoid) tumors," Alimentary Pharmacology \& Therapeutics, vol. 31, no. 2, pp. 169-188, 2009.

[34] J. Kang, C. Yoo, H.-S. Hwang et al., "Efficacy and safety of lanreotide in Korean patients with metastatic, well-differentiated gastroenteropancreatic-neuroendocrine tumors: a retrospective analysis," Investigational New Drugs, vol. 37, no. 4, pp. 763-770, 2019.

[35] H. Jann, T. Denecke, M. Koch, U. F. Pape, B. Wiedenmann, and M. Pavel, "Impact of octreotide long-acting release on tumour growth control as a first-line treatment in neuroendocrine tumours of pancreatic origin," Neuroendocrinology, vol. 98, no. 2, pp. 137-143, 2013.

[36] R. Arnold, M. E. Trautmann, W. Creutzfeldt et al., "Somatostatin analogue octreotide and inhibition of tumour growth in metastatic endocrine gastroenteropancreatic tumours," Gut, vol. 38, no. 3, pp. 430-438, 1996.

[37] C. Toumpanakis and M. E. Caplin, "Update on the role of somatostatin analogs for the treatment of patients with gastroenteropancreatic neuroendocrine tumors," Seminars in Oncology, vol. 40, no. 1, pp. 56-68, 2013.

[38] M. V. Davi', E. Cosaro, S. Piacentini et al., "Prognostic factors in ectopic cushing's syndrome due to neuroendocrine tumors: a multicenter study," European Journal of Endocrinology, vol. 176, no. 4, pp. 453-461, 2017.

[39] C. G. Genc, H. J. Klümpen, M. G. H. van Oijen, C. H. J. van Eijck, and E. J. M. Nieveen van Dijkum, "A nationwide population-based study on the survival of patients with pancreatic neuroendocrine tumors in The Netherlands," World Journal of Surgery, vol. 42, no. 2, pp. 490-497, 2018.

[40] G. Kaltsas, M. Caplin, P. Davies et al., "ENETS consensus guidelines for the standards of care in neuroendocrine tumors: pre- and perioperative therapy in patients with neuroendocrine tumors," Neuroendocrinology, vol. 105, no. 3, pp. $245-254,2017$. 